\title{
Creases on the interface between two soft materials
}

\section{Citation}

Jin, Lihua, Dayong Chen, Ryan C. Hayward, and Zhigang Suo. 2014. “Creases on the Interface Between Two Soft Materials." Soft Matter 10 (2): 303-311. doi:10.1039/c3sm51512e.

\section{Published Version}

doi:10.1039/C3SM51512E

\section{Permanent link}

http://nrs.harvard.edu/urn-3:HUL.InstRepos:25757883

\section{Terms of Use}

This article was downloaded from Harvard University's DASH repository, and is made available under the terms and conditions applicable to Open Access Policy Articles, as set forth at http:// nrs.harvard.edu/urn-3:HUL.InstRepos:dash.current.terms-of-use\#OAP

\section{Share Your Story}

The Harvard community has made this article openly available.

Please share how this access benefits you. Submit a story.

\section{Accessibility}




\title{
Creases on the interface between two soft materials
}

Lihua Jin, ${ }^{1}$ Dayong Chen, ${ }^{2}$ Ryan C. Hayward ${ }^{2, *}$, and Zhigang Suo, ${ }^{1, *}$

${ }^{1}$ School of Engineering and Applied Sciences, Kavli Institute for Nanobio Science and

Technology, Harvard University, Cambridge, Massachusetts 02138, USA

${ }^{2}$ Department of Polymer Science and Engineering, University of Massachusetts, Amherst, Massachusetts 01003, USA

\begin{abstract}
Theory and experiment are presented to show that an interface between two soft materials under compression can form creases, a type of bifurcation distinct from wrinkles. While creases bifurcate from a state of flat interface by a deformation localized in space and large in amplitude, wrinkles bifurcate from a state of flat interface by a deformation nonlocal in space and small in amplitude. The interfacial creases set in at a lower critical compression than interfacial wrinkles, but higher than surface creases. The condition for the onset of interfacial creases is scale-free, and is calculated in terms of elastic moduli, pre-strains and applied strains.
\end{abstract}

\footnotetext{
*rhayward@mail.pse.umass.edu, suo@seas.harvard.edu
} 


\section{Introduction}

Bonded materials with dissimilar properties are ubiquitous. They exist in composite materials ${ }^{1}$, geological strata ${ }^{2,3}$, electronic devices ${ }^{4}$, biological tissues ${ }^{5,6}$ and soft actuators ${ }^{7}$. In many cases, stresses arise due to differences in thermal expansion ${ }^{8-10}$, chemical reactions ${ }^{11}$, growth ${ }^{5,12}$, swelling ${ }^{13}$, or mechanical pre-strains ${ }^{14}$. Such mismatches may cause buckling instabilities ${ }^{1,10,15,16}$, which can reduce the strength of structures ${ }^{1,10,15,16}$ and lead to debonding of the interfaces $8,9,17$.

Interfacial instability has long been regarded as a bifurcation from a state of flat interface 1, 10, 15, 16. For example, Biot ${ }^{18,19}$ studied two elastic materials compressed along a direction parallel to the interface. He predicted that the interface forms wrinkles at a critical compression, similar to the formation of wrinkles at the free surface of a soft solid ${ }^{20}$. These wrinkles have dynamic analogs: Stoneley waves on the interface between two materials ${ }^{18,21-23}$, and Rayleigh waves on the surface of a homogeneous material 21, 24, 25. Wrinkles, however, have not been observed on surfaces of homogeneous elastic materials under compression; rather, creases have been observed ${ }^{13}, 26-29$. A recent theory shows that surface wrinkles and surface creases are two types of instability, and the critical strain for the onset of surface wrinkles is higher than that for the onset of surface creases ${ }^{30}$.

Motivated by this recent understanding, here we reconsider the stability of the interface between two materials. Our theory and experiment show an analogous localized, largeamplitude instability, which we call interfacial creases (Fig. 1). Wrinkles and creases are two types of bifurcation from the state of flat interface. While wrinkles bifurcate by a deformation nonlocal in space and small in amplitude, creases bifurcate by a deformation local in space and large in amplitude. In this regard, the wrinkling instability is of the same type as buckling of columns and plates, while the creasing instability is reminiscent of cracking, cavitation, shear banding, and phase transformation. Upon initiation, an interfacial crease corresponds to a field scaled by a single length scale. The critical strain is independent of this length scale and is scale- 
free. We calculate the critical conditions for the onset of the interfacial creases and find that, just like their surface counterparts, interfacial creases always form at lower compression than interfacial wrinkles. We show that interfacial creases are V-shaped. The presence of two materials prevents the interface from self-contact, but the tip of an interfacial crease is singular. Because the interface does not self-contact, the analysis of interfacial creases is simplified compared to that of surface creases. We demonstrate the existence of interfacial creases by experiments with hydrogels. By using differential swelling of two types of gels, we can form interfacial creases and surface creases independently.

\section{Analysis and simulations}

\subsection{Interfacial creases under applied strain}

We study the initiation of interfacial creases by comparing two states of equilibrium subject to the same applied strain (Fig. 1). In the state of flat interface, the field in either material is homogeneous, and the elastic energy of the state is denoted by $U_{0}$. In the creased state, one point of the interface is prescribed with a downward displacement $L$, and the elastic energy of the state is denoted $U$. The magnitude of the displacement $L$ is arbitrary, so long as it is much smaller than any other length in the boundary-value problem (e.g., the thickness and width of the block used in computation). Once $L$ is prescribed, the creased state is determined by a boundary-value problem of finite deformation. The two states of equilibrium differ by a deformation of large amplitude, but the difference is localized in a small region scaled by the length $L$. The two materials are taken to be incompressible and neo-Hookean, with shear moduli $G_{A}$ and $G_{B}$. We begin with the case that pre-strains are absent, and the block is compressed to reduce its width under plane strain conditions. The applied strain $\varepsilon$ is defined as the reduction in the width of the block divided by the width of the block in the undeformed state. Dimensional considerations dictate that the energy of the creased state should differ from that of the flat state by 


$$
U-U_{0}=G_{B} L^{2} f\left(\varepsilon, G_{B} / G_{A}\right) .
$$

As indicated, the dimensionless number $f$ is a function of the applied strain and the ratio of the shear moduli. The scaling relation (1) generalizes the result for surface creases ${ }^{31}$.

Without loss of generality, we assume that $G_{A}<G_{B}$. We expect that interfacial creases form by growing into the stiffer material (B). In the absence of applied strain, the energy of the state of flat interface vanishes, but the energy of the creased state is positive, so that the state of flat interface is stable. When a sufficiently large compressive strain is applied, the crease elongates the interface, so that the crease reduces energy in material $\mathrm{B}$, but increases energy in material A. Because $G_{A}<G_{B}$, the crease can reduce the total elastic energy in the two materials. For a fixed ratio $G_{B} / G_{A}$, the function $f\left(\varepsilon, G_{B} / G_{A}\right)$ decreases monotonically with the applied strain $\varepsilon$. An interfacial crease sets in when the creased state has the same energy as the flat state, namely,

$$
f\left(\varepsilon, G_{B} / G_{A}\right)=\mathrm{O} .
$$

This condition gives the critical strain for the onset of interfacial crease, $\varepsilon_{c}$, as a function of the modulus ratio, $G_{B} / G_{A}$.

The critical condition (2) for the onset of interfacial crease is independent of any length scale. This scale-free behavior results from two considerations. First, the prescribed displacement $L$ is assumed to be small compared to other lengths in the boundary-value problem, such as the thickness and width of the block used in the calculation. The flat state differs from the creased state by a deformation localized within a region scaled with $L$. Consequently, only the length $L$ enters (1), and no length appears in the critical condition (2). Second, the theory of elasticity does not have any material-defined length scale. The situation would be different if we accounted for the interfacial energy $\gamma$ between the two materials. The interfacial energy, together with an elastic modulus $G$, would provide a length scale, the 
elastocapillary length $\gamma / G$. The tip of an interfacial crease would be rounded on a length scale of $\gamma / G$, rather than being infinitely sharp. The interfacial energy would also provide a barrier against the initiation of interfacial creases, just as in the case of surface creases ${ }^{32}$. However, the elastocapillary length $\gamma / G$ is typically on the nanometer scale, and the energy barrier is correspondingly small. Thus the interfacial energy is ignored in this work.

In the above description, the creased state is determined by a boundary-value problem of finite deformation, and the prescribed displacement $L$ specifies the length scale. This particular way of specifying a length scale, however, does not affect the critical strain for the onset of the crease. Upon initiation, the creased state is scaled by one length, independent of how the length is specified.

We use the finite element software ABAQUS to solve the boundary-value problem of creased state, from the reference state of flat interface to the current state of a prescribed displacement $L$ (Fig. 2). Analysis of this type has also been used in our previous work on surface creases ${ }^{31}$. Due to symmetry, only a half of a crease is simulated. The materials deform under plane-strain conditions, and element type $\mathrm{CPE} 4 \mathrm{H}$ is used. Compare the two states subject to the same applied strain $\varepsilon$. Let $U_{\mathrm{o}}$ be the energy in the state of flat interface, and $U$ be the energy in the creased state. Their difference is plotted as a function of the applied strain $\varepsilon$ for a fixed value of the modulus ratio $G_{B} / G_{A}=5$. When $\varepsilon<0.512$, the state of flat interface has a lower elastic energy than the creased state. When $\varepsilon>0.512$, the state of flat interface has a higher elastic energy than the creased state. The strain $\varepsilon_{c}=0.512$ is identified as the critical condition for the onset of interfacial creases.

We use another finite element method to simulate the growth of an interfacial crease (Fig. 3). In the reference state, two materials A and B are bonded with a flat interface (Fig. 3(a)). We make use of the symmetry condition, and only simulate the right half of a crease. After a crease initiates, its growth does depend on the size of the block used in the calculation. Here the 
two materials in the reference state have the same thickness $H$, and the lateral size of the simulation box (corresponding to half of the spacing between creases) is set to be $W=H$, close to what is observed in our experiments. To seed the crease, a small defect is prescribed on the left end of the interface. The size of the defect is much smaller than the thickness of either material, but is large compared to the size of the surrounding mesh. An image of a region in the vicinity of the defect, with depth $D$ and angle $\alpha$, is shown in Fig. 3(b). In the current state, a horizontal displacement is applied on the boundary on the right (Fig. 3(c)). The top and bottom boundaries are constrained to remain planar, so that they cannot form surface creases. The boundary on the left is a plane of mirror symmetry. The two materials are compressed in the horizontal direction and expand in the vertical direction, while the depth of the crease, $L$, evolves as a function of the applied displacement.

The depth of the crease is plotted as a function of the applied strain (Fig. 4). The depth of the crease remains nearly zero below the critical strain, and begins to grow when the applied strain is beyond the critical value about $\varepsilon_{c}=0.512$. The crease is seeded with defects of different depths $D$ and angles $\alpha$. Meshes with different size are used as well: mesh 1 uses 18 elements along the defect; mesh 2 uses 36 elements along the defect. So long as a defect is small compared to the thickness of each material, but large compared to the size of surrounding mesh, the depth and the angle of the defect and the mesh size have a negligible effect on the critical strain for the onset of the crease and subsequent growth of the crease.

The elastic energy is as a function of the applied strain is (Fig. 5). Let $U_{0}$ be the energy in the two materials in the state of flat interface, and $U_{F}$ be the energy in the two materials calculated by using the finite element method of simulating crease growth. When the applied strain $\varepsilon<0.512$, the interface remains flat, and the total elastic energy is the same as that in the two materials in the state of flat interface $U_{F} / U_{0}=1$. When $\varepsilon>0.512$, a crease forms, reducing the total elastic energy below that in the two materials in the state of flat interface, $U_{F} / U_{0}<1$. 
Consequently, we identify $\varepsilon=0.512$ as the critical strain for the onset of creases, which is consistent with the result by the method of prescribing a length scale (Fig. 2). After an interfacial crease forms, the elastic energy of the bottom material $U_{B}$ decreases while the elastic energy of the top material $U_{A}$ increases. Since the bottom material is stiffer than the top material, the decrease in elastic energy of the bottom material is larger than the increase in the energy of the top material. Consequently, the interfacial crease reduces the total energy $U_{F}$.

Similarly, we can simulate the initiation and growth of interfacial creases for other modulus ratios. As expected, the critical strain for the onset of interfacial crease decreases as the modulus ratio $G_{B} / G_{A}$ increases (Fig. 6(a)). In the limit $G_{B} / G_{A} \rightarrow \infty$, the critical strain for the onset of an interfacial crease recovers that of a surface crease, $\varepsilon_{c}=0.354{ }^{30,}{ }^{31}$. In the limit $G_{B} / G_{A} \rightarrow 1$, the two materials are elastically homogeneous, and an interfacial crease will not form under any compressive strain, so that $\varepsilon_{c} \rightarrow 1$. Also plotted in Fig. 6(a) is the critical strain for the onset of interfacial wrinkles. While the onset of interfacial creases is determined by a boundary-value problem of finite deformation, the onset of interfacial wrinkles is determined by a boundary-value problem of infinitesimal deformation superimposed on a known finite deformation 18, 19; see Method and materials section. For any value of modulus ratio, the interfacial crease sets in at a critical strain lower than the interfacial wrinkles.

Fig. 6(b) shows the evolution of interfacial crease depth with the applied strain for different modulus ratios. The limit $G_{B} / G_{A} \rightarrow \infty$ corresponds to a surface crease. At a lower value of $G_{B} / G_{A}$, material A retards the onset of the crease, and the interfacial crease is shallower than the surface crease under the same applied strain. The calculated shapes of the creased interfaces are V-shaped (Fig. 6(c)). Under the same strain $\varepsilon=0.55$, the interfacial 
crease is deeper for a larger value of $G_{B} / G_{A}$, and asymptotically approaches the shape of a surface crease with self-contact as $G_{B} / G_{A} \rightarrow \infty$.

\subsection{Interfacial creases under both applied strain and pre-strains}

We next study interfacial creases under both pre-strains and applied strains (Fig. 7). Prior to bonding, material $\mathrm{B}$ is subject to pre-stretches $\lambda_{1}^{\text {pre }}$ and $\lambda_{2}^{\text {pre }}$, while material $\mathrm{A}$ is undeformed (Fig. 7 (a), (b)). The pre-stretches are defined as the current length divided by the original length, with subscripts 1 and 2 indicating the coordinate directions in the plane of the interface. After bonding, both materials are subject to applied stretches $\lambda_{1}$ and $\lambda_{2}$, which can be controlled independently of the pre-stretches $\lambda_{1}^{\text {pre }}$ and $\lambda_{2}^{\text {pre }}$ (Fig. 7 (c)). Both materials are taken to be incompressible, $\lambda_{1}^{\text {pre }} \lambda_{2}^{\text {pre }} \lambda_{3}^{\text {pre }}=1$ and $\lambda_{1} \lambda_{2} \lambda_{3}=1$.

For the same reason stated above, the energy in the creased state differs from that in the flat state by $U-U_{\mathrm{o}}=G_{B} L^{2} f$, but $f$ now depends on five dimensionless parameters: $\lambda_{1}, \lambda_{2}, \lambda_{1}^{\text {pre }}, \lambda_{2}^{\text {pre }}, G_{B} / G_{A}$. A further analysis, however, indicates that the critical condition for the onset of interfacial creases depends on three combined parameters:

$$
f\left(\lambda_{1} \sqrt{\lambda_{2}}, \lambda_{1}^{\text {pre }} \sqrt{\lambda_{2}^{\text {pre }}}, G_{B} / G_{A} \lambda_{2}^{\text {pre }}\right)=0 .
$$

This result is obtained as follows.

In analyzing creases, we assume that the materials deform under the generalized plane strain conditions (Fig. $7(\mathrm{c})$ ). The deformation gradient is invariant with respect to axis 2 and takes the form

$$
\mathbf{F}=\left[\begin{array}{ccc}
F_{11} & 0 & F_{13} \\
0 & \lambda_{2} & 0 \\
F_{31} & 0 & F_{33}
\end{array}\right]
$$


The free energy density of material A is

$$
W_{A}=\frac{G_{A}}{2} F_{i K} F_{i K}+\frac{G_{A}}{2} \lambda_{2}^{2} .
$$

Each repeated index implies a sum over 1 and 3. Consider an intermediate state in which material $\mathrm{A}$ is stretched along the three axes by $\left(\lambda_{2}^{-1 / 2}, \lambda_{2}, \lambda_{2}^{-1 / 2}\right)$. Using this intermediate state as a new reference state, the deformation gradient in the plane of axes 1 and 3 is $\hat{F}_{i K}=\lambda_{2}^{1 / 2} F_{i K}$. Rewrite the free energy density as

$$
W_{A}=\frac{G_{A}}{2 \lambda_{2}} \hat{F}_{i K} \hat{F}_{i K}+\frac{G_{A}}{2} \lambda_{2}^{2}
$$

The free energy density in material $\mathrm{B}$ is

$$
W_{B}=\frac{G_{B}}{2} F_{i K} F_{K \alpha}^{p r e} F_{i L} F_{L \alpha}^{p r e}+\frac{G_{B}}{2}\left(\lambda_{2} \lambda_{2}^{\text {pre }}\right)^{2}
$$

Write $\hat{F}_{K \alpha}^{\text {pre }}=\left(\lambda_{2}^{\text {pre }}\right)^{1 / 2} F_{K \alpha}^{\text {pre }}$, so that

$$
W_{B}=\frac{G_{B}}{2 \lambda_{2} \lambda_{2}^{\text {pre }}} \hat{F}_{i K} \hat{F}_{K \alpha}^{\text {pre }} \hat{F}_{i L} \hat{F}_{L \alpha}^{\text {pre }}+\frac{G_{B}}{2}\left(\lambda_{2} \lambda_{2}^{\text {pre }}\right)^{2} .
$$

Because $\lambda_{2}$ and $\lambda_{2}^{\text {pre }}$ are prescribed constants, the last terms in Eqs. (6) and (8) do not contribute to the energy difference between the creased state and the flat state. The first terms in Eqs. (6) and (8) describe the energy change from the intermediate states to the current states, with plane strain deformation $\hat{F}_{i K}$ in material A and plane strain deformation and pre-stretches $\hat{F}_{i K} \hat{F}_{K \alpha}^{p r e}$ in material B. Consequently, the deformation under generalized plane strain conditions correspond to the deformation under plane strain conditions, with the effective shear moduli $G_{A} / \lambda_{2}$ and $G_{B} / \lambda_{2} \lambda_{2}^{p r e}$ for the two materials. Thus we show that the critical condition for the onset of creases depends on three dimensionless parameters: $\lambda_{1} \sqrt{\lambda_{2}}, \lambda_{1}^{\text {pre }} \sqrt{\lambda_{2}^{\text {pre }}}, G_{B} / G_{A} \lambda_{2}^{\text {pre }}$. This result generalizes the critical condition for the onset of surface creases ${ }^{31}$. The same conclusion 
holds for the critical condition for the onset of wrinkles. Similar combinations of parameters have been identified in an independent analysis of wrinkles in film-substrate structures 33 .

The critical condition for the onset of interfacial creases is calculated using finite element method. We write a user-defined material subroutine UMAT to include the pre-stretches in the free energy function. For a given value of $G_{B} / G_{A} \lambda_{2}^{\text {pre }}$, the critical condition is represented by a curve in the plane spanned by parameters $\lambda_{1} \sqrt{\lambda_{2}}$ and $\lambda_{1}^{\text {pre }} \sqrt{\lambda_{2}^{\text {pre }}}$ (Fig. 8(a)). Below the curve interfacial creases form, and above the curve interfacial creases do not form. The condition for the onset of surface creases corresponds to the limit $G_{B} / G_{A} \lambda_{2}^{\text {pre }} \rightarrow \infty$. The critical condition for the onset of wrinkles is plotted in a similar diagram (Fig. 8(b)). For a given value of $G_{B} / G_{A} \lambda_{2}^{\text {pre }}$, the curve for interfacial creases always lies above the one for interfacial wrinkles. This result predicts that interfacial creases will form, rather than interfacial wrinkles.

\section{Experiment results}

We perform experiments to show the existence of interfacial creases. In one experiment, two hydrogels are confined between glass slides (Fig. 9). The two gels are bonded to each other, but are not bonded to the glass slides. The colored gel is stiffer than the colorless gel. The former has a polymer network containing fixed charges, while the latter has an uncharged network (Fig. 9(a)). After preparation, the two gels are nearly stress-free, and the interface is flat (Fig. 9(b)). When placed in water and allowed to swell, the charged gel is subject to compressive stress due to its inability to expand laterally, leading to the formation of interfacial creases (Fig. 9(c)). The uncharged gel swells negligibly, so that the top surface does not form surface creases.

A second experiment is performed to compare interfacial creases and surface creases (Fig. 10). A charged gel of thickness $H_{2}=6-8 \mu \mathrm{m}$ is attached on a glass substrate. A portion of the gel is then covered with an uncharged gel of thickness $H_{1}=76 \mu \mathrm{m}$ (Fig. 10 (a)). When immersed in a phosphate buffered saline solution containing $274 \mathrm{mM}$ sodium chloride, both 
gels swell slightly and generate in-plane equibiaxial compressive stresses, but the compression is insufficient to cause either surface or interfacial creases. Deionized water is then added drop by drop to reduce the concentration of the saline solution, so that the charged gel swells more and the uncharged gel swells negligibly (as characterized by fluorescence confocal microscopy). When the swelling ratio of the charged gel reaches $H_{2}^{\prime} / H_{2}=2.4 \pm 0.1$, the uncovered portion of the charged gel forms surface creases, while the interface between the two gels remains flat (Fig. 10 (b), (d)). This critical swelling ratio for the onset of surface creases is in good agreement with previous reports ${ }^{29}$. When the swelling ratio is increased to $H_{2}^{\prime \prime} / H_{2}=3.3 \pm 0.1$, the covered region of the gel forms interfacial creases (Fig. 10 (c), (e)). At the same degree of swelling, the interfacial creases are shallower than surface creases, as shown by the cross-sectional confocal images in the insets of Fig. 10(e). Both surface and interfacial creases show a spacing of $\sim 20 \mu \mathrm{m}$,

or roughly 2.5 -3 times $H_{2}$. The gel of uncharged network swells negligibly, and its surface remains flat.

Further details of the two experiments can be found in the Method and materials section. While these experimental observations qualitatively agree with our theoretical predictions, quantitative comparison between experiments and calculations would require that constitutive behavior of the gels be characterized with precision and used in the calculations. This task is beyond the scope of the present work.

\section{Linear perturbation analysis for the onset of interfacial wrinkles}

We calculate the critical condition for the onset of interfacial wrinkles by using the smallperturbation method described by Biot ${ }^{18,19}$. Additional results are obtained here to account for pre-stretches. The analysis relies on the theory of finite deformation of an elastic body. The reference state is taken to be the bonded state, where the bottom material is pre-stretched, while the top material is undeformed (Fig. 7(b)). Label a material particle in the body by its 
coordinate $\mathbf{X}$ when the body is in the reference state. In the deformed state, the material particle moves to a place of coordinate $\mathbf{x}$. The function $\mathbf{x}(\mathbf{X})$ describes the deformation of the body. The deformation gradient is

$$
F_{i K}=\frac{\partial x_{i}(\mathbf{X})}{\partial X_{K}} .
$$

The nominal stress $s_{i K}$ satisfies the conditions of equilibrium

$$
\frac{\partial s_{i K}}{\partial X_{K}}=0
$$

The interface between materials A and B coincides with the plane $X_{3}=0$. Across the interface, both the displacement and traction are continuous:

$$
\begin{aligned}
& x_{i}^{A}=x_{i}^{B}, \\
& s_{i_{3}}^{A}=s_{i_{3}}^{B} .
\end{aligned}
$$

Each material is characterized by the Helmholtz free energy as a function of the deformation gradient, $W(\mathbf{F})$. The nominal stress relates to the deformation gradient by

$$
s_{i K}=\frac{\partial W(\mathbf{F})}{\partial F_{i K}} .
$$

The materials are taken to be incompressible:

$$
\operatorname{det}(\mathbf{F})=\mathbf{1}
$$

The materials are described by the neo-Hookean model. For material A, the free energy is

$$
W_{A}(\mathbf{F})=\frac{G_{A}}{2} F_{i K} F_{i K}-(\operatorname{det} \mathbf{F}-1) \Pi,
$$

where $\Pi$ is the Lagrange multiplier used to enforce incompressibility. In general the Lagrange multiplier is inhomogeneous, and should be solved as a part of the problem. The material model Eq. (13) becomes 


$$
s_{i K}^{A}=G_{A} F_{i K}-\Pi H_{i K}
$$

where $\mathbf{H}=\mathbf{F}^{-T}$. In the reference state (Fig. 7(b)), material B is pre-stretched to a homogeneous state with deformation gradient:

$$
\mathbf{F}^{\text {pre }}=\left[\begin{array}{ccc}
\lambda_{1}^{\text {pre }} & 0 & 0 \\
0 & \lambda_{2}^{\text {pre }} & 0 \\
0 & 0 & \lambda_{3}^{\text {pre }}
\end{array}\right]
$$

The material $\mathrm{B}$ is also taken to be incompressible, $\operatorname{det} \mathbf{F}^{\text {pre }}=\lambda_{1}^{\text {pre }} \lambda_{2}^{\text {pre }} \lambda_{3}^{\text {pre }}=1$. For material $\mathrm{B}$, the net deformation gradient between the current state and the undeformed state is $F_{i K} F_{K \alpha}^{p r e}$, and the free energy is

$$
W_{B}=\frac{G_{B}}{2} F_{i K} F_{K \alpha}^{p r e} F_{i L} F_{L \alpha}^{p r e}-(\operatorname{det} \mathbf{F}-1) \Pi
$$

The material model Eq. (13) becomes

$$
s_{i K}^{B}=G_{B} F_{K \alpha}^{p r e} F_{L \alpha}^{p r e} F_{i L}-\Pi H_{i K} \cdot
$$

We next perturb from the state of finite deformation (Fig. $7(\mathrm{c})$ ), of displacement $x_{i}^{0}(\mathbf{X})$, deformation gradient $F_{i K}^{0}(\mathbf{X})$ and nominal stress $s_{i K}^{0}(\mathbf{X})$. In particular, the deformation gradient of this state is prescribed by the applied stretches:

$$
\mathbf{F}^{0}=\left[\begin{array}{ccc}
\lambda_{1} & 0 & 0 \\
0 & \lambda_{2} & 0 \\
0 & 0 & \lambda_{3}
\end{array}\right]
$$

The corresponding field of displacement is $\mathbf{x}^{0}=\mathbf{F}^{0} \mathbf{X}$. This state of finite deformation is then perturbed by a state of infinitesimal deformation $\tilde{\mathbf{x}}(\mathbf{X})$. We write the perturbed state as $\mathbf{x}=\mathbf{x}^{0}(\mathbf{X})+\widetilde{\mathbf{x}}(\mathbf{X})$. The other fields are similarly perturbed. Both state $\mathbf{x}^{0}(\mathbf{X})$ and the state $\mathbf{x}^{0}(\mathbf{X})+\widetilde{\mathbf{x}}(\mathbf{X})$ satisfy the same governing equations (9)-(19). Eqs (9)-(12) reduce to equations for the fields of perturbation: 


$$
\begin{gathered}
\tilde{F}_{i K}=\frac{\partial \tilde{x}_{i}(\mathbf{X})}{\partial X_{K}}, \\
\frac{\partial \widetilde{s}_{i K}}{\partial X_{K}}=0, \\
\tilde{x}_{i}^{A}=\widetilde{x}_{i}^{B}, \\
\widetilde{s}_{i 3}^{A}=\widetilde{s}_{i 3}^{B} .
\end{gathered}
$$

The condition of incompressibility Eq. (14) is perturbed as

$$
H_{i K}^{0} \widetilde{F}_{i K}=0
$$

where $\mathbf{H}^{0}=\mathbf{F}^{0^{-\mathbf{T}}}$. Expanding Eq. (16) in the Taylor series at $\mathbf{F}^{0}$ and keeping only the linear term, we obtain that

$$
\widetilde{S}_{i K}^{A}=\left(G_{A} \delta_{i j} \delta_{K L}+\Pi^{0} H_{i L}^{0} H_{j K}^{0}\right) \widetilde{F}_{j L}-H_{i K}^{0} \widetilde{\Pi}
$$

Similarly perturbing Eq. (19), we obtain that

$$
\widetilde{s}_{i K}^{B}=\left(G_{B} F_{K \alpha}^{p r e} F_{L \alpha}^{p r e} \delta_{i j}+\Pi^{0} H_{i L}^{0} H_{j K}^{0}\right) \widetilde{F}_{j L}-H_{i K}^{0} \widetilde{\Pi} .
$$

Inserting Eqs. (21), (26) and (27) into Eq. (22), we obtain that

$$
\begin{gathered}
\left(G_{A} \delta_{i j} \delta_{K L}+\Pi^{0} H_{i L}^{0} H_{j K}^{0}\right) \frac{\partial^{2} \widetilde{x}_{j}(\mathbf{X})}{\partial X_{K} \partial X_{L}}-H_{i K}^{0} \frac{\partial \widetilde{\Pi}(\mathbf{X})}{\partial X_{K}}=0, \\
\left(G_{B} F_{K \alpha}^{p r e} F_{L \alpha}^{p r e} \delta_{i j}+\Pi^{0} H_{i L}^{0} H_{j K}^{0}\right) \frac{\partial^{2} \widetilde{x}_{j}(\mathbf{X})}{\partial X_{K} \partial X_{L}}-H_{i K}^{0} \frac{\partial \widetilde{\Pi}(\mathbf{X})}{\partial X_{K}}=0,
\end{gathered}
$$

for material A and B respectively. For a given field $x_{i}^{0}(\mathbf{X})$, Eqs (25), (28), and (29) define a set of linear, homogeneous partial differential equations for the fields of perturbation, $\tilde{x}_{i}(\mathbf{X})$ and $\tilde{\Pi}(\mathbf{X})$. The displacement and the traction are continuous across the interface. Consequently, the fields of perturbation $\tilde{x}_{i}(\mathbf{X})$ and $\tilde{\Pi}(\mathbf{X})$ are governed by an eigenvalue problem. The characteristic equation of this eigenvalue problem defines the critical condition for the onset of 
wrinkles. Following an analysis similar to that of Biot ${ }^{18,19}$, we obtain the critical condition for the onset of interfacial wrinkles:

$$
\begin{aligned}
& {\left[\frac{\lambda_{2}^{\text {pre }} G_{A}}{G_{B}}\left(\frac{\lambda_{3}}{\lambda_{1}}+1\right)+\left(\frac{\lambda_{3} \lambda_{3}^{\text {pre }}}{\lambda_{1} \lambda_{1}^{\text {pre }}}+1\right)\right]\left[\frac{\lambda_{2}^{\text {pre }} G_{A}}{G_{B}}\left(\frac{\lambda_{1}}{\lambda_{3}}+1\right)+\left(\frac{\lambda_{1} \lambda_{1}^{\text {pre }}}{\lambda_{3} \lambda_{3}^{\text {pre }}}+1\right)\right]} \\
& -\left[\frac{\lambda_{2}^{\text {pre }} G_{A}}{G_{B}}\left(1-\frac{\lambda_{3}}{\lambda_{1}}\right)-\left(1-\frac{\lambda_{3} \lambda_{3}^{\text {pre }}}{\lambda_{1} \lambda_{1}^{\text {pre }}}\right)\right]^{2}=0 .
\end{aligned}
$$

This condition is plotted in Fig. 8(b). When $\lambda_{1}^{\text {pre }}=\lambda_{2}^{\text {pre }}=\lambda_{3}^{\text {pre }}=1$, the condition recovers Biot's solution ${ }^{18,19}$, and is plotted in Fig. 6(a).

\section{Experimental methods}

\subsection{Experiment with hydrogels confined in a glass slit}

A glass slit, thickness $1 \mathrm{~mm}$ and width $28 \mathrm{~mm}$, is constructed by gluing glass slides together (Fig. 9). The inner surfaces of the slit are treated with trimethylchlorosilane to reduce adhesion of the gels to the glass surfaces. A charged gel layer is first prepared by loading the slit with an aqueous pre-gel solution containing $700 \mathrm{mM} \mathrm{N}$-isopropyl-acrylamide, $660 \mathrm{mM}$ sodium acrylate and $45 \mathrm{mM} \mathrm{N,N'-methylenebisacrylamide.} \mathrm{Gelation} \mathrm{is} \mathrm{initiated} \mathrm{by} \mathrm{adding} 1 \mu \mathrm{L}$ of $10 \%$ w/w ammonium persulfate aqueous solution as the initiator and $0.3 \mu \mathrm{L} N, N, N^{\prime}, N^{\prime}-$ tetramethylenediamine as the catalyst into $200 \mu \mathrm{L}$ of freshly degassed pre-gel solution immediately prior to loading the chamber. To stain the charged gel, a small amount $\left(3.2 \times 10^{-5}\right.$ mmol) of a Rhodamine B-labeled methacrylate is incorporated into the $200 \mu \mathrm{L}$ pre-gel solution. Next, a softer uncharged gel is prepared on top of the charged gel using the same procedure as above, but with a pre-gel solution containing $701 \mathrm{mM}$ acrylamide and $19 \mathrm{mM} N, N^{\prime}-$ methylenebisacrylamide. Some interpenetration of the second gel into the existing charged gel does occur, and helps to bond the materials together, but based on the relatively rapid speed of gelation $(\sim 20 \mathrm{~min})$, this length-scale is small compared to the $\sim 1 \mathrm{~cm}$ dimensions of the gels. The contrast in modulus between the gels is not known, but based simply on the relative 
crosslinker concentrations, the charged gel should be at least $\sim 2.4$-fold stiffer than the uncharged gel 34. Following gelation, this confined gel sample is soaked in deionized water for 3 weeks to reach swelling equilibrium, and the shape of the interface is recorded using a digital camera.

\subsection{Experiment with thin layers of gels on a glass substrate}

This experiment uses a photo-crosslinkable poly( $N$-isopropylacrylamide-co-sodium acrylate-co-acrylamidobenzophenone) polymer described in previous reports ${ }^{29,35}$. A glass cover slip substrate is pretreated with [3-(methacryloxy)-propyl]trichlorosilane to provide covalently anchoring, then a copolymer film is solution cast from chloroform to yield a film thickness of $H_{2}=6-8 \mu m$, followed by crosslinking by exposure to $365 \mathrm{~nm}$ UV light. A much softer uncharged gel is next formed by loading a freshly prepared de-gassed aqueous pre-gel solution

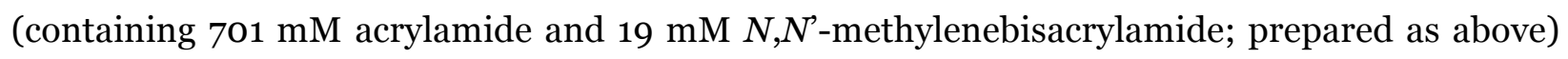
between the substrate-attached photo-crosslinked polymer film and a clean cover slip, and allowing $20 \mathrm{~min}$ for polymerization to proceed. The uncharged gel film thickness $H_{1}=76 \mu \mathrm{m}$ is defined using Kapton $300 \mathrm{HN}$ films (DuPont) as spacers. To prevent swelling of the photocrosslinked poly( $N$-isopropylacrylamide) film by the pre-gel solution, the neutral gel is formed at $70{ }^{\circ} \mathrm{C}$. Following polymerization, the cell is cooled to room temperature and the glass slides are separated. The surface-attached gel bilayer is then transferred to a phosphate buffered saline swelling solution containing $274 \mathrm{mM}$ sodium chloride. Deionized water is then added drop-wise to dilute the swelling solution and thus increase the swelling ratio of the charged gel, while the formation of creases is monitored in situ using either an upright optical microscope (Zeiss Axiotech Vario) in reflected-light differential interference contrast mode or a laser scanning confocal fluorescence microscope (Zeiss). Based on a previous report, we estimate the Young's modulus of the uncharged gel to be $\sim 5 \mathrm{kPa}{ }^{36}$, while that of the charged gel is estimated to be several hundred $\mathrm{kPa}$ based on the density of photo-crosslinking groups in the copolymer. 


\section{Conclusion}

We present calculations and experiments to show that an interface between two soft materials under compression can form creases. A creased state deviates from a state of flat interface by a deformation large in amplitude and local in space. The critical condition for the onset of interfacial creases is scale-free. This critical condition is calculated in terms of elastic moduli, pre-stretches and applied stretches by allowing deviation from a flat state with a large deformation. By contrast, a wrinkled state deviates from a state of flat interface by a deformation small in amplitude and nonlocal in space. The critical condition for the onset of interfacial wrinkles is calculated by a small-perturbation method. In all cases studied, the critical conditions for the onset of interfacial creases are reached before those for the onset of interfacial wrinkles.

\section{Acknowledgements}

The work at Harvard is supported by the National Science Foundation through MRSEC (DMR-0820484) and by the U.S. Army Research Office through MURI (W911NF-09-1-0476). The work at UMass is supported by the NSF through grant DMR-0747756, with additional funding from the MRSEC on Polymers at UMass (DMR-0820506). 


\section{References}

1. C. Mueller, A. Gorius, S. Nazarenko, A. Hiltner and E. Baer, J Compos Mater, 1996, 30, 1912-1921.

2. $\quad$ G. B. Brietzke, A. Cochard and H. Igel, Geophys $J$ Int, 2009, 178, 921-938.

3. $\quad$ A. N. Guz and V. N. Chekhov, Int Appl Mech, 2007, 43, 127-159.

4. $\quad$ Y. G. Sun and J. A. Rogers, Adv Mater, 2007, 19, 1897-1916.

5. $\quad$ M. Basan, J. F. Joanny, J. Prost and T. Risler, Phys Rev Lett, 2011, 106, 158101.

6. $\quad$ P. B. Noble, A. R. West, R. A. McLaughlin, J. J. Armstrong, S. Becker, P. K. McFawn, J. P. Williamson, P. R. Eastwood, D. R. Hillman, D. D. Sampson and H. W. Mitchell, J Appl Physiol, 2010, 108, 401-411.

7. $\quad$ I. Tokarev and S. Minko, Soft Matter, 2009, 5, 511-524.

8. J. S. Wang and A. G. Evans, Acta Mater, 1999, 47, 699-710.

9. D. R. Mumm, A. G. Evans and I. T. Spitsberg, Acta Mater, 2001, 49, 2329-2340.

10. $\quad$ S. H. Im and R. Huang, Acta Mater, 2004, 52, 3707-3719.

11. K. J. Zhao, M. Pharr, Q. Wan, W. L. Wang, E. Kaxiras, J. J. Vlassak and Z. G. Suo, J Electrochem Soc, 2012, 159, A238-A243.

12. T. Savin, N. A. Kurpios, A. E. Shyer, P. Florescu, H. Y. Liang, L. Mahadevan and C. J. Tabin, Nature, 2011, 476, 57-63.

13. T. Tanaka, S. T. Sun, Y. Hirokawa, S. Katayama, J. Kucera, Y. Hirose and T. Amiya, Nature, 1987, 325, 796-798.

14. J. S. Huang, J. Liu, B. Kroll, K. Bertoldi and D. R. Clarke, Soft Matter, 2012, 8, 62916300.

15. $\quad$ M. I. Darby and V. N. Kanellopoulos, J Phys D Appl Phys, 1987, 20, 298-302.

16. Y. Hu, A. Hiltner and E. Baer, Polym Composite, 2004, 25, 653-661.

17. S. R. Choi, J. W. Hutchinson and A. G. Evans, Mech Mater, 1999, 31, 431-447.

18. $\quad$ M. A. Biot, Proc. R. Soc. London, Ser. A, 1963, 273, 340-344.

19. $\quad$ M. A. Biot, $Q J$ Mech Appl Math, 1964, 17, 17-22.

20. M. A. Biot, Appl. Sci. Res., 1963, 12, 168-182.

21. D. M. Barnett, Int J Solids Struct, 2000, 37, 45-54.

22. D. M. Barnett, J. Lothe, S. D. Gavazza and M. J. P. Musgrave, Proc. R. Soc. London, Ser. $A, 1985$, 402, $153-166$.

23. $\quad$ R. Stoneley, Proc. R. Soc. London, Ser. A, 1924, 106, 416-428.

24. $\quad$ L. Rayleigh, $P$ Lond Math Soc, 1885, 1, 4-11.

25. K. Worden, Strain, 2001, 37, 167-172.

26. S. Q. Cai, D. Y. Chen, Z. G. Suo and R. C. Hayward, Soft Matter, 2012, 8, 1301-1304.

27. $\quad$ A. N. Gent and I. S. Cho, Rubber Chem Technol, 1999, 72, 253-262.

28. A. Ghatak and A. L. Das, Phys Rev Lett, 2007, 99, 076101.

29. V. Trujillo, J. Kim and R. C. Hayward, Soft Matter, 2008, 4, 564-569.

30. $\quad$ E. Hohlfeld and L. Mahadevan, Phys Rev Lett, 2011, 106, 105702.

31. W. Hong, X. H. Zhao and Z. G. Suo, Appl Phys Lett, 2009, 95, 111901.

32. $\quad$ D. Y. Chen, S. Q. Cai, Z. G. Suo and R. C. Hayward, Phys Rev Lett, 2012, 109, 038001.

33. J. W. Hutchinson, Philos T Roy Soc A, 2013, 371, 20120422.

34. S. P. Obukhov, M. Rubinstein and R. H. Colby, Macromolecules, 1994, 27, 3191-3198.

35. J. Kim, J. A. Hanna, M. Byun, C. D. Santangelo and R. C. Hayward, Science, 2012, 335, 1201-1205.

36. E. Sultan and A. Boudaoud, J Appl Mech-t Asme, 2008, 75, 051002. 


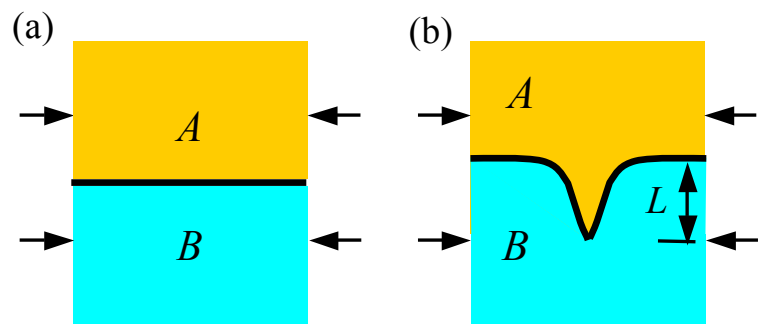

Fig. 1. Materials A and B are bonded at a flat interface. Subject to a compressive strain, the interface may either remain flat (a), or form creases (b). 


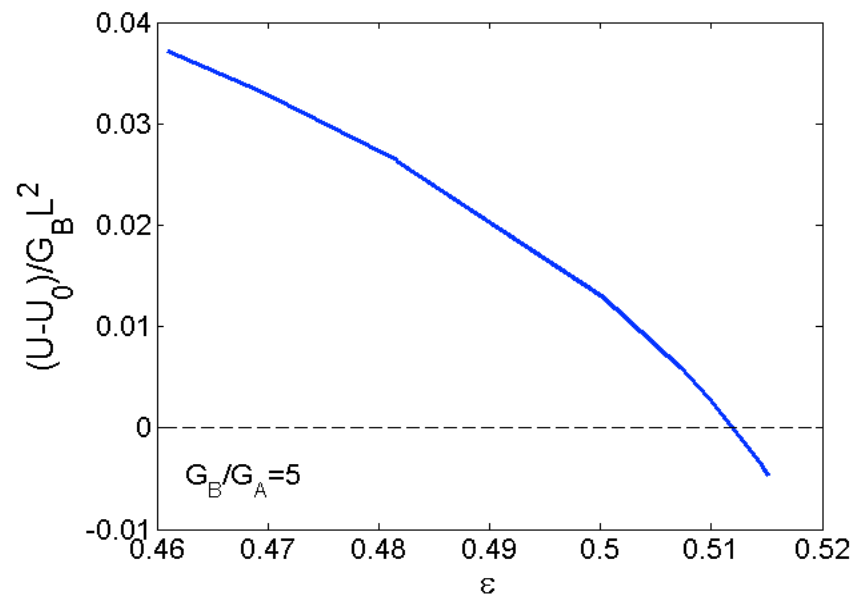

Fig. 2. The energy difference between the creased state $U$ and the flat state $U_{0}$ plotted as a function of the applied strain $\varepsilon$ for a fixed value of the modulus ratio $G_{B} / G_{A}=5$. When $\varepsilon=0.512$, the energy of the creased state is equal to the energy of the flat state, and $\varepsilon=0.512$ is identified as the critical condition for the onset of interfacial creases. 


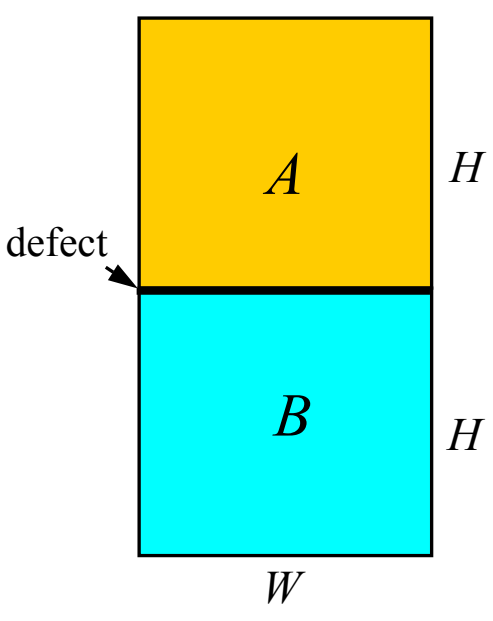

(a) Reference state

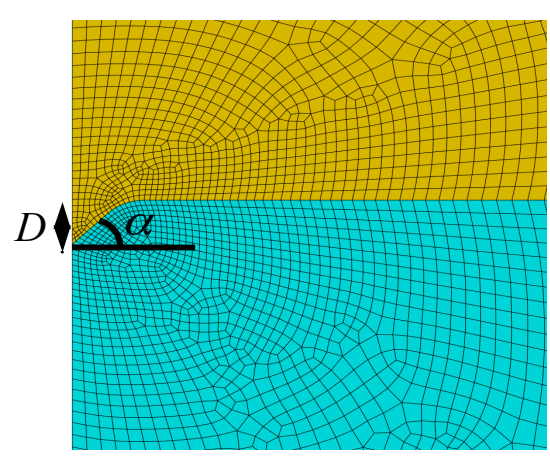

(b) Close-up of defect

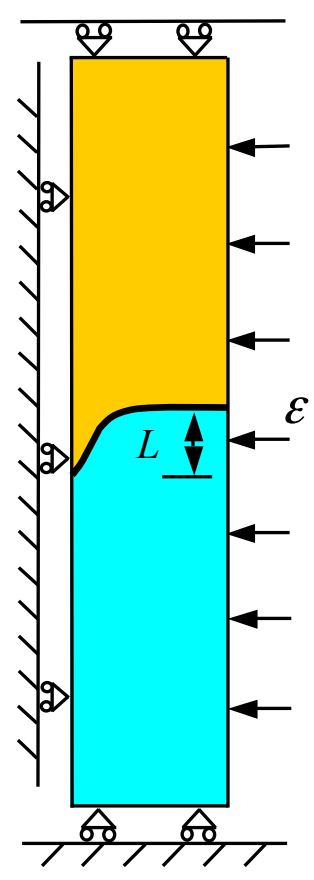

(c) Current state

Fig. 3. Finite element model. (a) In the reference state, two materials A and B are bonded with a flat interface. A small defect is prescribed on the left end of the interface. (b) An image of a region in the vicinity of the defect, with depth $D$ and angle $\alpha$. (c) In the current state, the two materials are compressed in the horizontal direction and expand in the vertical direction, while the depth of the crease, $L$, evolves as a function of the applied displacement. 


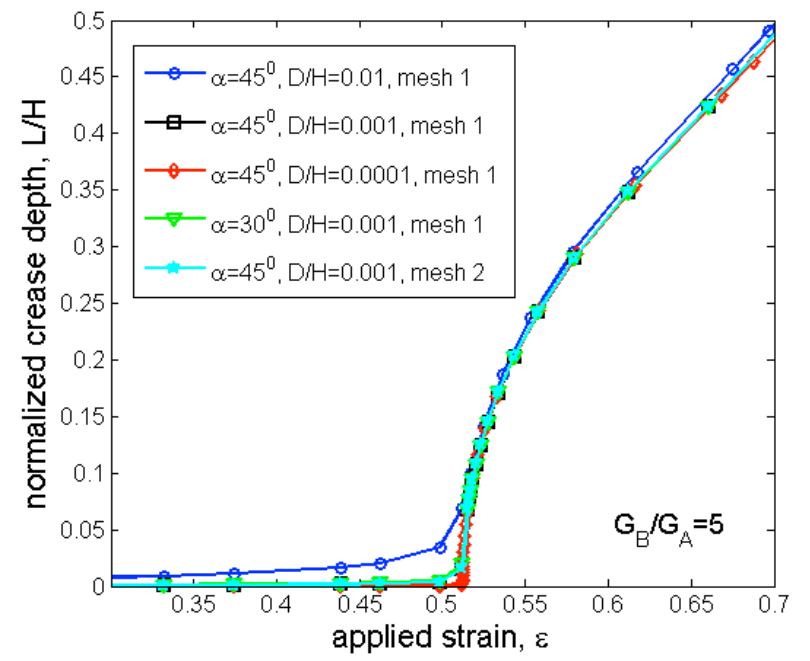

Fig. 4. The depth of the crease is plotted as a function of the applied strain. The crease is seeded with defects of different depths $D$ and angles $\alpha$. Mesh 1 uses 18 elements along the defect, and mesh 2 uses 36 elements along the defect. 

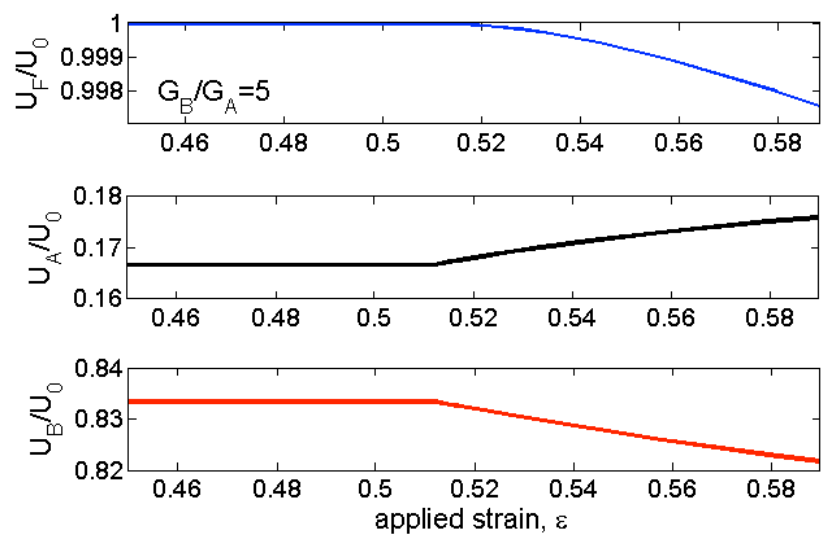

Fig. 5. Energy in both materials, $U_{F}$, energy in material A, $U_{A}$, and energy in material $\mathrm{B}, U_{B}$ are plotted as functions of the applied strain. Each energy is normalized by the energy in the two materials in the state of flat interface, $U_{0}$. 


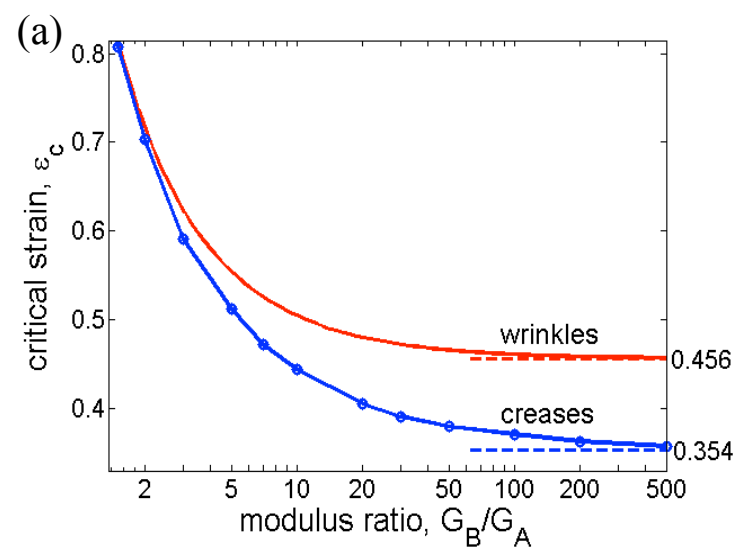

(b)
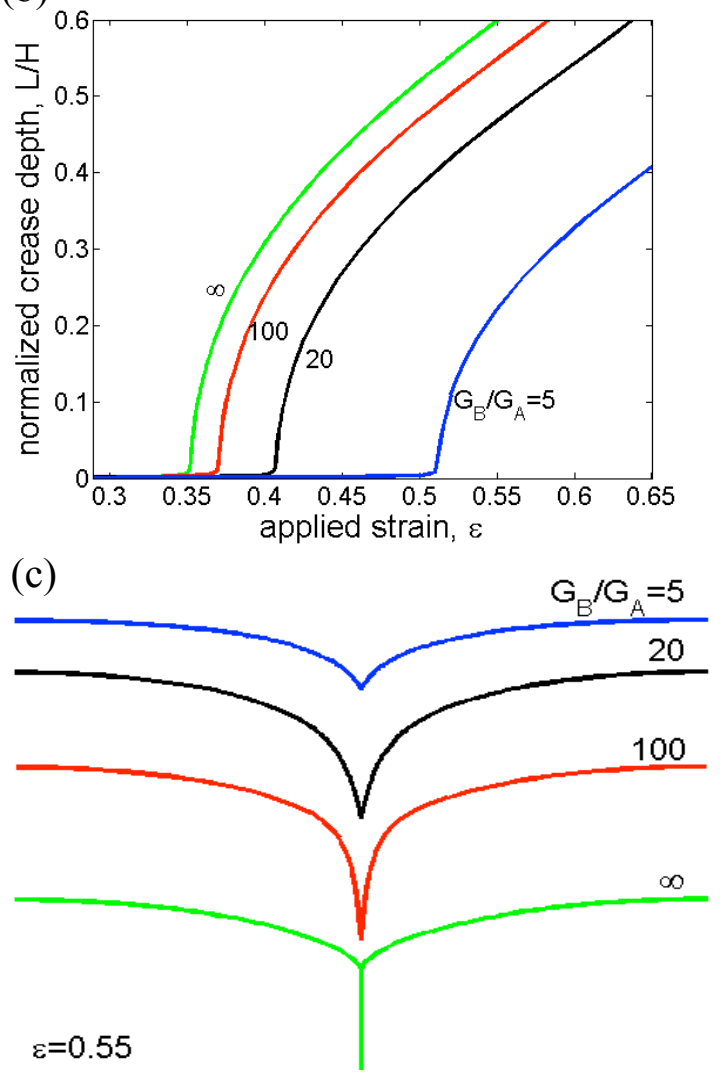

Fig. 6. Interfacial creases under applied strain. (a) The critical strain for the onset of creases as a function of the ratio of the shear modulus, $G_{B} / G_{A}$. Also plotted is the critical strain for the onset of wrinkles. (b) The depth of a crease as a function of the applied strain for several values of $G_{B} / G_{A}$. (c) Shapes of creased interfaces at applied strain $\varepsilon=0.55$, for several values of the modulus ratio. 

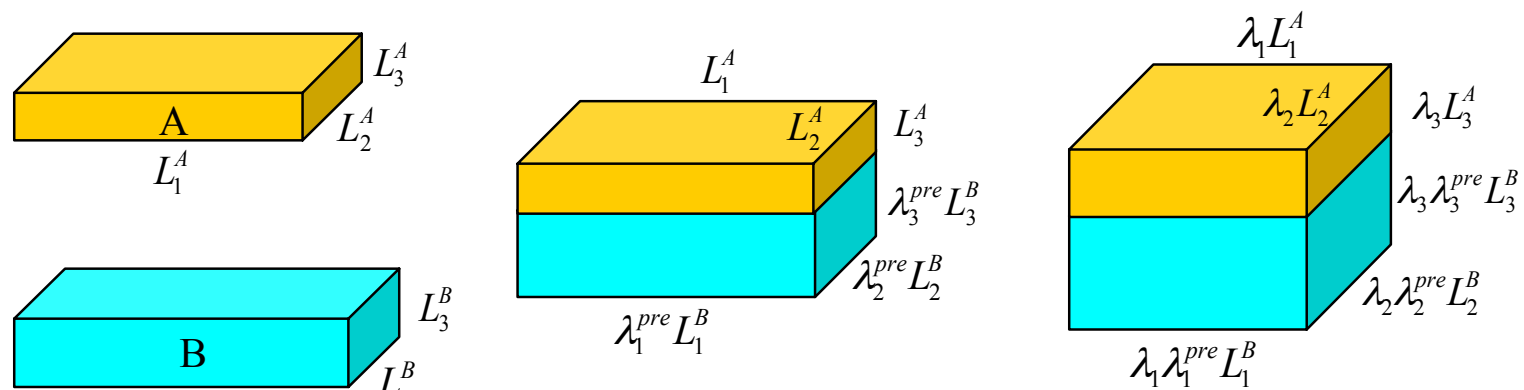

(a) Unbonded state

(b) Bonded state

(c) Loaded state

Fig. 7. Two materials with bonding on the interface under both pre-strains and applied strains. (a) In the unbonded state, both materials are stress-free. (b) In the bonded state, material B is subject to pre-stretches $\lambda_{1}^{\text {pre }}, \lambda_{2}^{\text {pre }}$ and $\lambda_{3}^{\text {pre }}$, and material A is stress-free. (c) In the loaded state, the bilayer is subject to applied stretches $\lambda_{1}, \lambda_{2}$ and $\lambda_{3}$. 
(a)

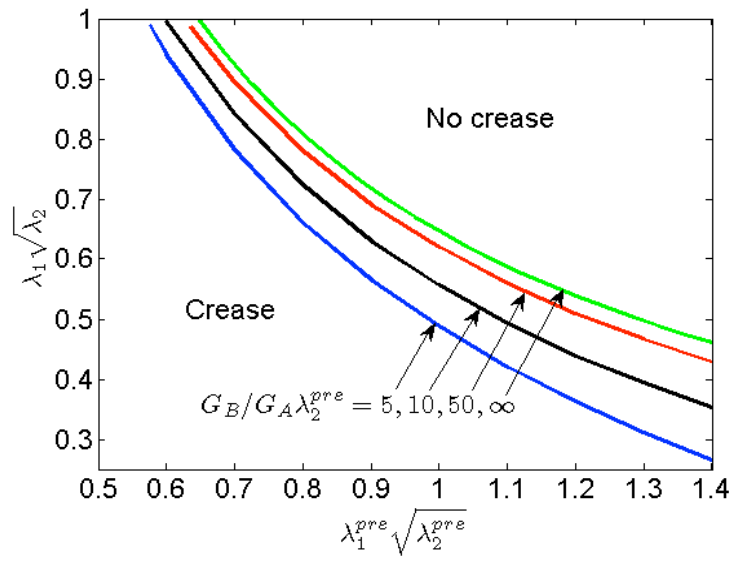

(b)

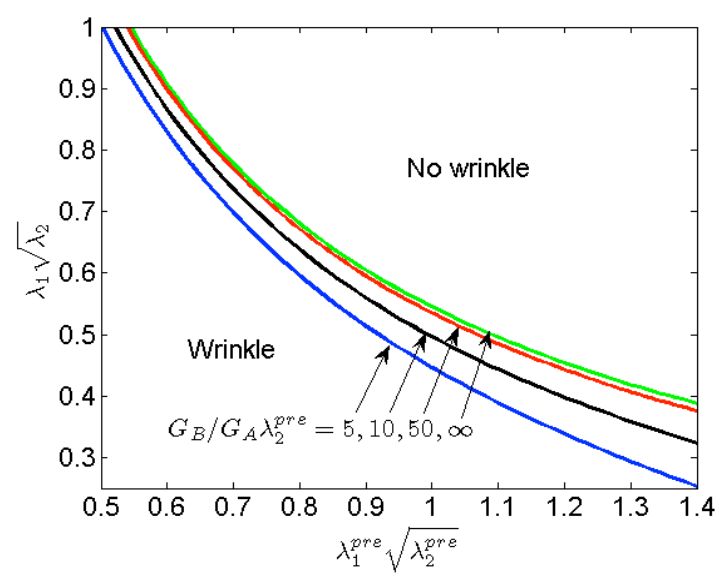

Fig. 8. (a) The critical condition for the onset of interfacial creases. (b) The critical condition for the onset of interfacial wrinkles. 

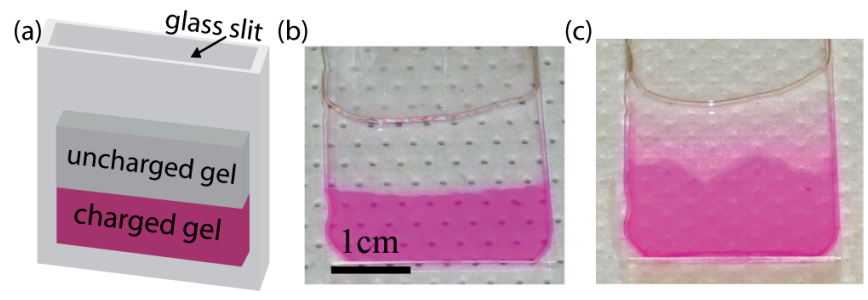

Fig. 9. Creases formed on the interface between two hydrogels. (a) A schematic of the experimental setup. Confined in a $1 \mathrm{~mm}$ thick glass slit are two gels, one with a charged network and the other with unchanged network. The gels are bonded to each other, but are not bonded to the glass. (b) After the preparation, the two hydrogels are relatively dry and stress-free, and the interface is flat. (c) When soaked in water, the charged gel swells much more than the uncharged gel, so that creases form on the interface between the two gels, but not on the free surface of the uncharged gel. 


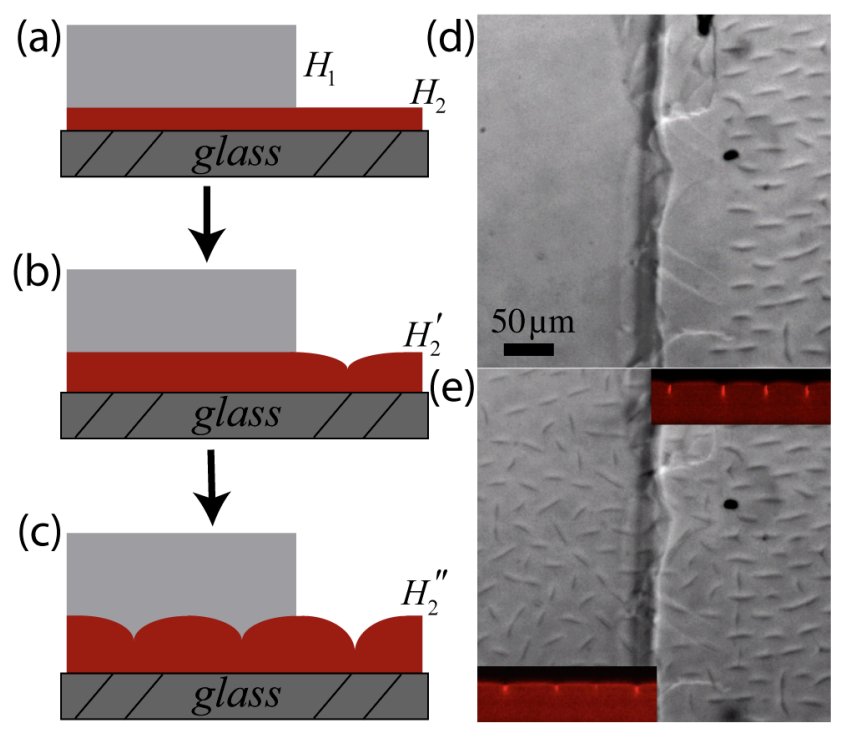

Fig. 10. A comparison of surface creases and interfacial creases. (a) A charged gel is attached to a glass substrate, and is partially covered by an uncharged gel. (b) Cross-sectional schematic and (d) top-view micrograph when the charged gel swells by $H_{2}{ }^{\prime} / H_{2}=2.4$. Creases form on the free surface of the charged gel, while the interface between the gels remains flat. (c) Cross-sectional schematic and (e) top-view micrograph when the charged gel swells by $H_{2}{ }^{\prime \prime} / H_{2}=3 \cdot 3$. Creases also form on the interface. The creases on the interface have a smaller depth than the creases on the surface, as shown by the cross-sectional confocal images in the insets. 